\title{
Trends in the Isobaric Multiplet Mass Equation Coefficients
}

\author{
Marion MacCormick ${ }^{1, a}$ and Georges Audi ${ }^{2}$ \\ ${ }^{1}$ Institut de Physique Nucléaire, CNRS/IN2P3, Université Paris-Sud, 91406 Orsay CEDEX, France \\ ${ }^{2}$ Centre de Spectrométrie Nucléaire et de Spectrométrie de Masse, CNRS/IN2P3, Université Paris-Sud, \\ Bât. 108, F-91405 Orsay Campus, France
}

\begin{abstract}
Isobaric analogue states (IAS) can be used to study the charge independence of the nuclear force via first order perturbation theory. In this case the IAS multiplet masses are expected to follow a quadratic form as described by the Isobaric Multiplet Mass Equation (IMME) with coefficients accessible through experimental measurements. Higher order effects are expected to appear through cubic, or higher, polynomial terms. The current IMME coefficient trends, as based on the IAS states included in the 2012 Atomic Mass Evaluation and NUBASE2012 are shown.
\end{abstract}

\section{Introduction}

A mass relationship can be observed in isobars belonging to the same isospin multiplet around $N=Z$ and for masses $A<60$. The ground state of a given nuclide may be identified as an excited state in the multiplet members. The full set of member states, known as isobaric analogue states (IAS) have, by definition, the same spin-parity and isospin. The main difference between their masses can be attributed to the charge symmetry of the nucleon-nucleon interaction [1] and so these differences may be used to explore the charge symmetry and charge independence of the nuclear interaction.

The mass of nuclides around $N=Z$, with atomic mass number $A$ and $A=N+Z$, can be considered to arise from a charge independent strong interaction with an additional charge dependent Coulomb component due to the interaction between protons [2]. The isospin projection $T_{z}$ for a given nuclide is written

$$
T_{z}=\frac{N-Z}{2}
$$

where $N$ and $Z$ are the neutron and proton number, respectively. The members of a given isospin multiplet $T$ have projections $T_{z}$ running from $-T$ to $+T$. Internal isospin states $T$, describing the analogue ground state of the most neutron-rich nuclide, can take the values

$$
\left|\frac{N-Z}{2}\right| \leq T \leq \frac{N+Z}{2}
$$

The mass $M$ can then be written as

$$
M\left(A, T, T_{z}\right)=M_{0}+E_{C} T_{z}+\Delta_{n H} T_{z}
$$

\footnotetext{
ae-mail: maccorm@ipno.in2p3.fr
} 
$\Delta_{n H}$ being the neutron- ${ }^{1} \mathrm{H}$ mass difference, $E_{c}$ the Coulomb energy and $M_{0}$ the charge-free nuclear mass [3]. In this paper we focus only on the analogues of ground states.

A simplified Coulomb energy description is obtained, as given in equation 4 , where $E_{C}^{(0)}, E_{C}^{(1)}$ and $E_{C}^{(2)}$ are isoscalar, isovector and isotensor Coulomb energies, respectively.

$$
\begin{aligned}
E_{C}\left(A, T, T_{z}\right) & =E_{C}^{(0)}(A, T) \\
& -T_{Z} E_{C}^{(1)}(A, T) \\
& +\left[3 T_{z}^{2}-T(T+1)\right] E_{C}^{(2)}(A, T)
\end{aligned}
$$

It is then possible to write the isobaric multiplet mass equation (IMME) [3, 5], for a given isobar, in a quadratic form as shown in equation 5

$$
M\left(T, T_{z}\right)=a+b T_{z}+c T_{z}^{2}
$$

where the coefficients $a, b$ and $c$ are related to the Coulomb energy with $a=M_{0}+E_{C}^{(0)}-T(T+1) E_{C}^{(2)}$, $b=\Delta_{n H}+E_{C}^{(1)}$ and $c=3 E_{C}^{(2)}$.

The current trends in the IMME coefficients, $a, b$ and $c$, based on the recent AME2012 [6, 7] ground state masses and NUBASE2012 [8] excited states are shown along with higher order $d$ coefficients.

\section{Second order IMME}

Experiments giving access to data on mass multiplets up to $A \sim 60$ and isospins $T=\frac{1}{2}$ to $T=3$ have been studied in detail. The IMME second order polynomial coefficients $a, b$ and $c$ have been extracted and are shown in figures 1 to 3 . The dataset for the $T=\frac{5}{2}$ multiplets covers the range $A=11$

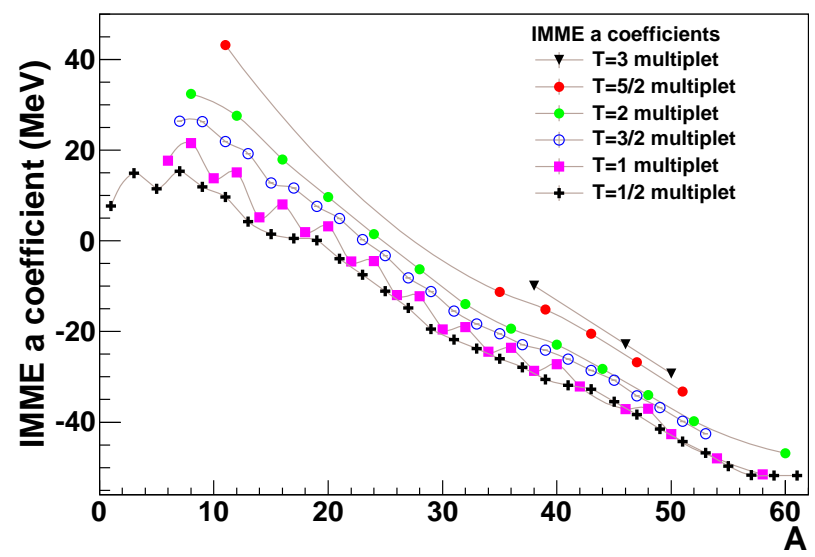

Figure 1. $a$ coefficients, in $\mathrm{MeV}$, shown as a function of atomic mass number $A$ extracted from a second degree IMME polynomial fit: $a+b T_{z}+c T_{z}^{2}$. This coefficient is the mass excess of the excited $N=Z$ nuclide for integer isospin values, and is more difficult to interpret theoretically. 
to 51 while the $T=3$ dataset is rather limited, with only three IAS mass multiplets experimentally established in the range $A=46$ to 58. In general only three out of the potential six or seven isobaric analogue states in each the multiplets have been observed, and so only a second order IMME fit can be applied to these datasets.

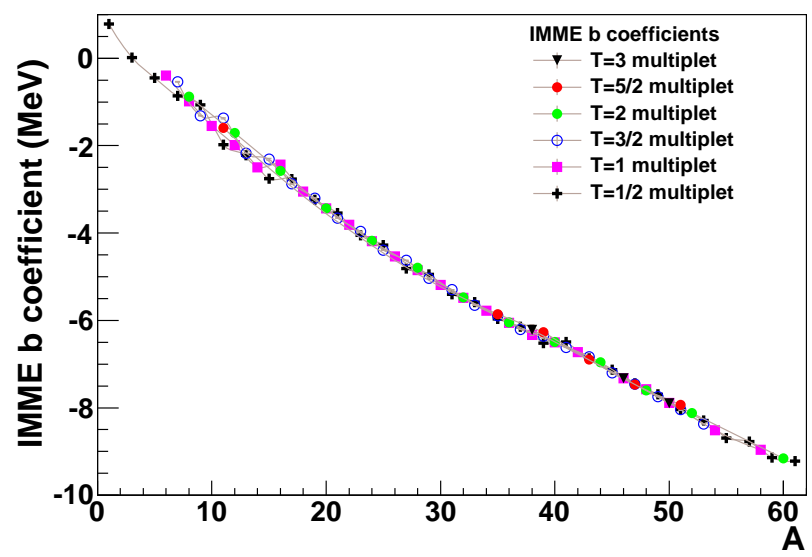

Figure 2. $b$ coefficients, in $\mathrm{MeV}$, shown as a function of atomic mass number $A$ extracted from a second degree IMME polynomial fit: $a+b T_{z}+c T_{z}^{2}$. This coefficient is interpreted as being directly related to the vector part of the Coulomb force $b=\Delta_{n H}+E_{C}^{(1)}$.

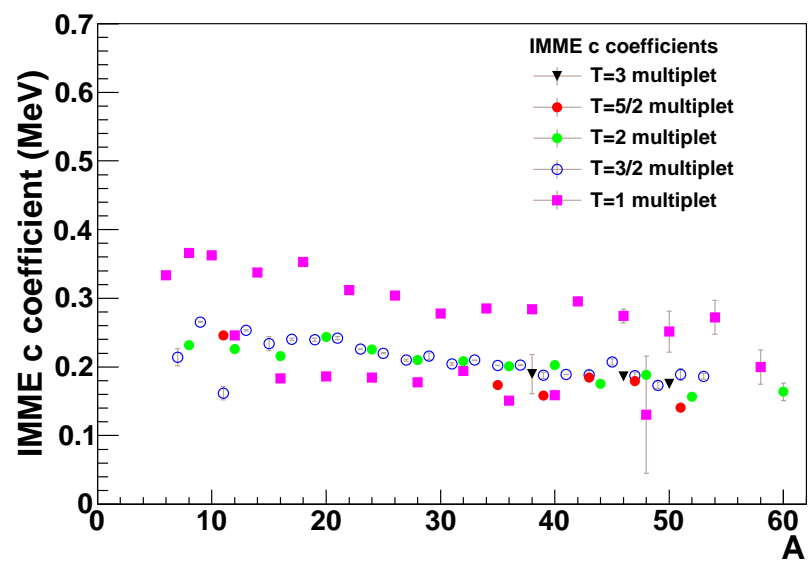

Figure 3. $c$ coefficients, in $\mathrm{MeV}$, shown as a function of atomic mass number $A$ extracted from a second degree IMME polynomial fit: $a+b T_{z}+c T_{z}^{2}$. This coefficient is interpreted as being directly related to the tensor part of the Coulomb force $c=3 E_{C}^{(2)}$. 


\section{Third order IMME}

If first order perturbation theory is sufficient to describe the IAS mass relationships, a second order IMME is sufficient. However, if higher order effects do intervene, each of the coefficients $a, b$ and $c$ may contain higher order terms [1]. One simple method to test for this is to fit the experimental multiplets with a third degree IMME and so giving rise to a $d$ term. Datasets with at least four experimentally established IAS were fitted to a third degree IMME: $\Delta M=a+b T_{z}=c T_{z}^{2}+d T_{z}^{3}$. The $a, b$ and $c$ coefficients should remain stable as compared to the second order IMME if the fit is a good estimate of the mass excesses. With the exception of the $A=11 T=\frac{3}{2}$ multiplet, and $A>40$ for $T=2$, where fragmented states have been observed, the other coefficients remained stable. The $d$ coefficients are shown in figure 4 and are found to be non-zero (in association with stable $a, b$ and $c$ coefficients) for $A=8,9,32,40$ and 53 and based on a $3 \sigma$ displacement from zero. This work is ongoing and a complete set of precise values is currently being prepared.

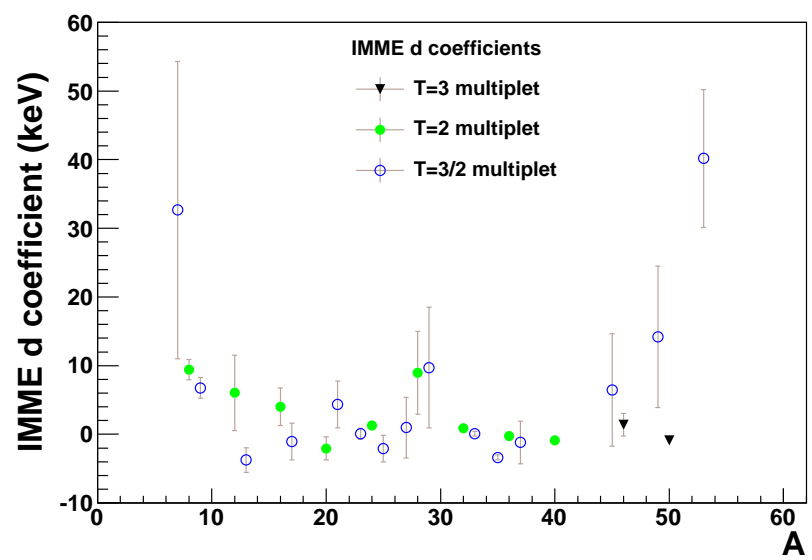

Figure 4. $d$ coefficients, in $\mathrm{keV}$, shown as a function of atomic mass number $A$ extracted from a third degree IMME polynomial fit: $a+b T_{z}+c T_{z}^{2}+d T_{z}^{3}$.

The authors are most grateful for access to detailed notes left by A. H. Wapstra (deceased Dec. 2006).

\section{References}

[1] E. P. Wigner. 1st Proceedings of the Robert A. Welch Foundation Conferences on Chemical Research, Houston (1957), Houston, Texas. I chapter IV, page 86 (1958).

[2] S. Weinberg, S. B. Treiman, Phys. Rev. 116, 465 (1959).

[3] D. H. Wilkinson, Phys. Lett. 13, 571 (1964).

[4] W. M. Macdonald, Phys. Rev. 100, 51 (1955).

[5] J. J.änecke, Phys. Rev. 147, 735 (1966).

[6] G. Audi, M. Wang, A. H. Wapstra, F. G. Kondev et al.,Chinese Physics C, 36, 1287-1602 (2012)

[7] M. Wang, G. Audi, A. H. Wapstra, F. G. Kondev et al.,Chinese Physics C, 36, 1603-2014 (2012)

[8] G. Audi, F. G. Kondev, M. Wang, B. Pfeiffer et al., Chinese Physics C, 36, 1157-1286 (2012). 